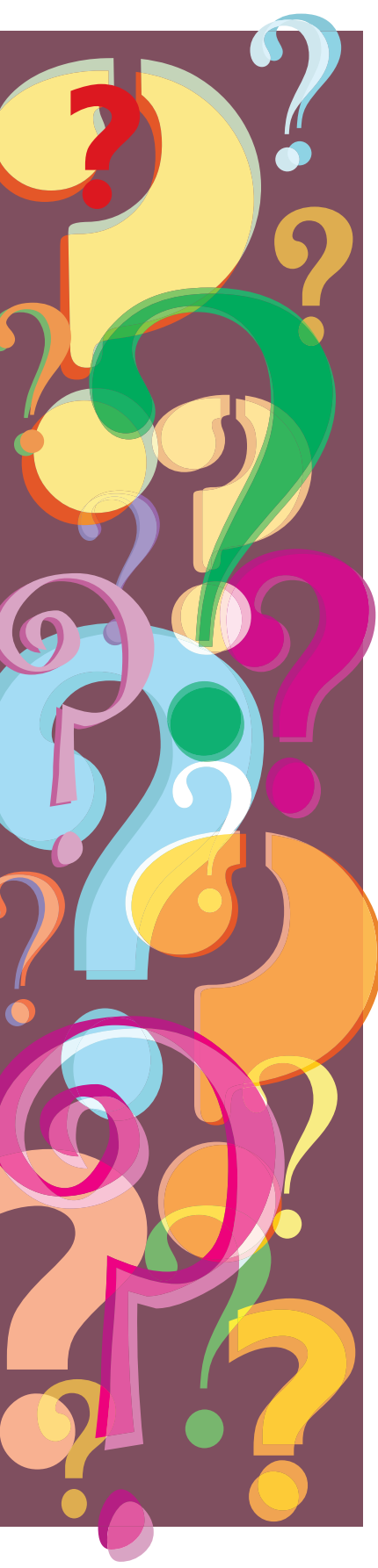

\title{
Do they or don't they?
}

The proposal that astrocytes modulate neuronal function by releasing neurotransmitters in a $\mathrm{Ca}^{2+}$-dependent manner suggests the possibility of an added layer of information processing in the brain. However, this idea has been highly controversial. In a recent study published in Science, Agulhon, Fiacco and McCarthy investigated the role of the astrocytic $G_{q} G$ protein-coupled receptor (GPCR) in the regulation of $\mathrm{Ca}^{2+}$-dependent gliotransmission in mouse hippocampal slices. They found no evidence that modifying astrocytic $\mathrm{Ca}^{2+}$ levels affects spontaneous or evoked excitatory transmission at Schaffer collateralCA1 synapses, thereby contradicting a host of other publications.

Several in situ (slices) and in vivo studies clearly showed that $\mathrm{Ca}^{2+}$ transients in mature astrocytes are physiologically driven by metabotropic $\mathrm{G}_{\mathrm{q}}$ GPCRs, which are activated by spillover of presynaptically released neurotransmitters. Astrocytic $\mathrm{G}_{\mathrm{q}}$ GPCRs are therefore considered the primary physiological link between neuronal activity and $\mathrm{Ca}^{2+}$ increases in astrocytes. Other studies in cultured astrocytes and brain slices showed that pharmacologically induced $\mathrm{Ca}^{2+}$ elevations in astrocytes trigger the release of gliotransmitters (glutamate, ATP or D-serine), modulating presynaptic and postsynaptic receptors and therefore synaptic transmission and plasticity. Taken together, these findings led to the proposal of gliotransmission. This concept is analogous to neurotransmission, whereby $\mathrm{Ca}^{2+}$ rises trigger the release of transmitters on a short time scale. A heated debate ensued regarding the functional relevance of gliotransmission in vivo.

To test the validity of the gliotransmission hypothesis, the authors investigated acute hippocampal slices derived from transgenic mice in which $\mathrm{G}_{\mathrm{q}}$ GPCR-triggered $\mathrm{Ca}^{2+}$ signalling could be selectively activated (in Mas-related GPCR member A1-expressing mice) or selectively inhibited (in inositol-1,4,5-trisphosphate receptor type 2 (ITPR2)knockout mice). In contrast to previous pharmacological approaches, these novel molecular tools enabled the authors to selectively and directly manipulate the physiological regulators of $\mathrm{Ca}^{2+}$ transients $\left(\mathrm{G}_{\mathrm{q}}\right.$ GPCRs and ITPR2) in mature passive astrocytes. In addition, they examined the effect of activating endogenous astrocytic $\mathrm{G}_{\mathrm{q}}$ GPCRs in wild-type slices. In a series of electrophysiological experiments, they assessed the presynaptic release probability of Schaffer collaterals, the spontaneous excitatory postsynaptic currents (EPSCs) and the NMDA ( $N$-methyl-D-aspartate) receptormediated component of evoked whole-cell EPSCs of CA1 pyramidal neurons, and the short-term plasticity, induction, magnitude and maintenance of long-term potentiation in CA1 neurons. They did not detect changes in these parameters when $\mathrm{Ca}^{2+}$ signalling through $\mathrm{G}_{\mathrm{q}}$ GPCRs was modulated in astrocytes.

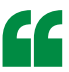

They found no evidence that modifying astrocytic $\mathrm{Ca}^{2+}$ levels affects spontaneous or evoked excitatory transmission

These findings suggest that changes in astrocytic intracellular $\mathrm{Ca}^{2+}$ levels as a result of the activation or inactivation of $\mathrm{G}_{\mathrm{q}}$ GPCRs or ITPR2 has no effect on synaptic function at neighbouring synapses. These results contrast with earlier studies that used pharmacological tools and imply that previous findings of gliotransmission might have been artefacts. Therefore, the physiological role of neuronally evoked $\mathrm{Ca}^{2+}$ transients in astrocytes remains controversial, calling for further investigation of this longstanding question.

Claudia Wiedemann

ORIGINAL RESEARCH PAPER Agulhon, C.,

Fiacco, T. A. \& McCarthy, K. D. Hippocampal short- and long-term plasticity are not modulated by astrocyte $\mathrm{Ca}^{2+}$ signaling. Science 327, 1250-1254 (2010)

FURTHER READING Hamilton, N. B. \& Attwell, D. Do astrocytes really exocytose neurotransmitters? Nature Rev. Neurosci. 11, 227-238 (2010) 